Bernhard Palme*

\title{
Arabische Papyri Online
}

DOI 10.1515/bfp-2016-0011

Zusammenfassung: Ein Schlüsselereignis der Weltgeschichte ist der plötzliche Aufstieg der Araber zur führenden Macht im 7.-8.Jh. n. Chr. Die historiographischen Quellen sind jedoch Jahrhunderte danach entstanden und projizieren spätere Verhältnisse auf die Zeit der Expansion. Zeitgenössischen Texten auf Papyrus kommt deshalb höchste Bedeutung zu. Das Forschungsvorhaben macht 15000 nicht publizierte Papyri mit wichtigen Informationen über das Früharabische Reich durch Digitalisierung zugänglich.

Schlüsselwörter: Papyri; Arabische Eroberung; Arabisches Reich; Digitalisierung

\section{Papyri of the Early Arab Empire Online}

Abstract: A key phenomenon in world history is the sudden rise of the Arabs to a leading power in the course of the 7th and 8th centuries $\mathrm{CE}$. While historiographic narratives date from later periods and have the tendency to project later conditions back onto the time of the conquest, contemporary texts preserved on papyrus are of crucial importance. The project sets out for digitizing 15,000 unpublished papyri, which provide information on the Early Arab Empire.

Keywords: Papyri; Arab conquest; Early Arab Empire; digitisation

Ein herausragendes Phänomen der Weltgeschichte ist der plötzliche Aufstieg der Araber zur führenden Weltmacht durch die Etablierung des Omayyadischen Reiches im Verlauf des 7. und frühen 8. Jh. n. Chr. Der rasche und überraschende Sieg über das Byzantinische Kaiserreich und die Zerstörung des Sassanidenreiches, die Expansion der arabischen Herrschaft von Persien bis Andalusien und die damit einhergehende Verbreitung des Islam haben nicht nur die politische und religiöse Landschaft des östlichen Mittelmeerraumes, Nordafrikas und des Nahen Ostens nachhaltig geprägt, sondern auch den Grundstein für politische, sprachliche und kulturelle Entwicklungen gelegt, die bis zum heutigen Tage wirken.

*Kontaktperson: Univ.-Prof. Dr. Bernhard Palme, bernhard.palme@onb.ac.at
Die historische Erforschung der Handlungsabläufe sowie der treibenden Kräfte hinter den politischen, militärischen, sozialen, wirtschaftlichen und religiösen Entwicklungen in den ersten Jahrhunderten des Arabischen Reiches steht jedoch vor einer komplizierten Quellenlage: Zeitgenössische Chroniken haben oft nur in Übersetzungen von zweifelhafter Verlässlichkeit überlebt, während byzantinische Geschichtsschreiber bloß am Rande auf die Ereignisse eingehen und koptische Märtyrergeschichten eine einseitige Sicht der Dinge präsentieren. Arabische Historiographen schildern die Phasen der Expansion zwar in lebendigen Erzählungen, sind aber erst viele Jahrzehnte oder sogar Jahrhunderte nach den Ereignissen entstanden. Sowohl die christlichen als auch die arabischen Quellen weisen daher die Tendenz auf, spätere Verhältnisse auf die Zeit der Expansion und Reichsgründung zu projizieren. Die ambivalenten Quellen haben zu lebhaften gelehrten Diskussionen über die frühe arabische Identität und Mentalität, über kulturelle und religiöse Wurzeln, Traditionen und Einflüssen geführt, so dass sogar in grundsätzlichen Fragen zur Frühgeschichte des Arabischen Reiches und der Ausbreitung des Islam bislang kein Konsens gefunden wurde.

Eine entscheidende Rolle in diesen Debatten sollte den zeitgenössischen Quellen urkundlichen Charakters zukommen. Neben den Werken der Geschichtsschreibung, die allesamt intentionale Überlieferung sind, bilden die Texte auf Papyrus, Pergament und frühem Papier einen zweiten, unabhängigen Strang von nicht-intentionalen Nachrichten. Eine Fülle solcher Schriftstücke aus der Phase der arabischen Expansion und Ausgestaltung des Herrschaftsbereiches hat sich vor allem in dem trockenen Klima Ägyptens erhalten. Diese Dokumente enthalten amtliche und private Korrespondenz, Urkunden von Rechtsgeschäften, Unterlagen der Buchhaltung und Steuererhebung, aber auch religiöse, magische und literarische Texte und beleuchten deshalb vielfältige Aspekte der damaligen Lebensumstände und Kultur. Entsprechend der multi-lingualen Gesellschaft des damaligen Ägyptens sind die relevanten Dokumente in Arabisch (seit der Mitte des 7.Jh.), Griechisch (bis zum Ende des 8. Jh.) und Koptisch (während des gesamten Zeitraumes) abgefasst. Während Griechisch und Koptisch die Landessprachen am Vorabend der arabischen Eroberung waren, gewann Arabisch erst in den Jahrzehnten nach der Eroberung Ägyptens (639-641 n. Chr.) allmählich an Boden. Für die Geschichte des Früh- 


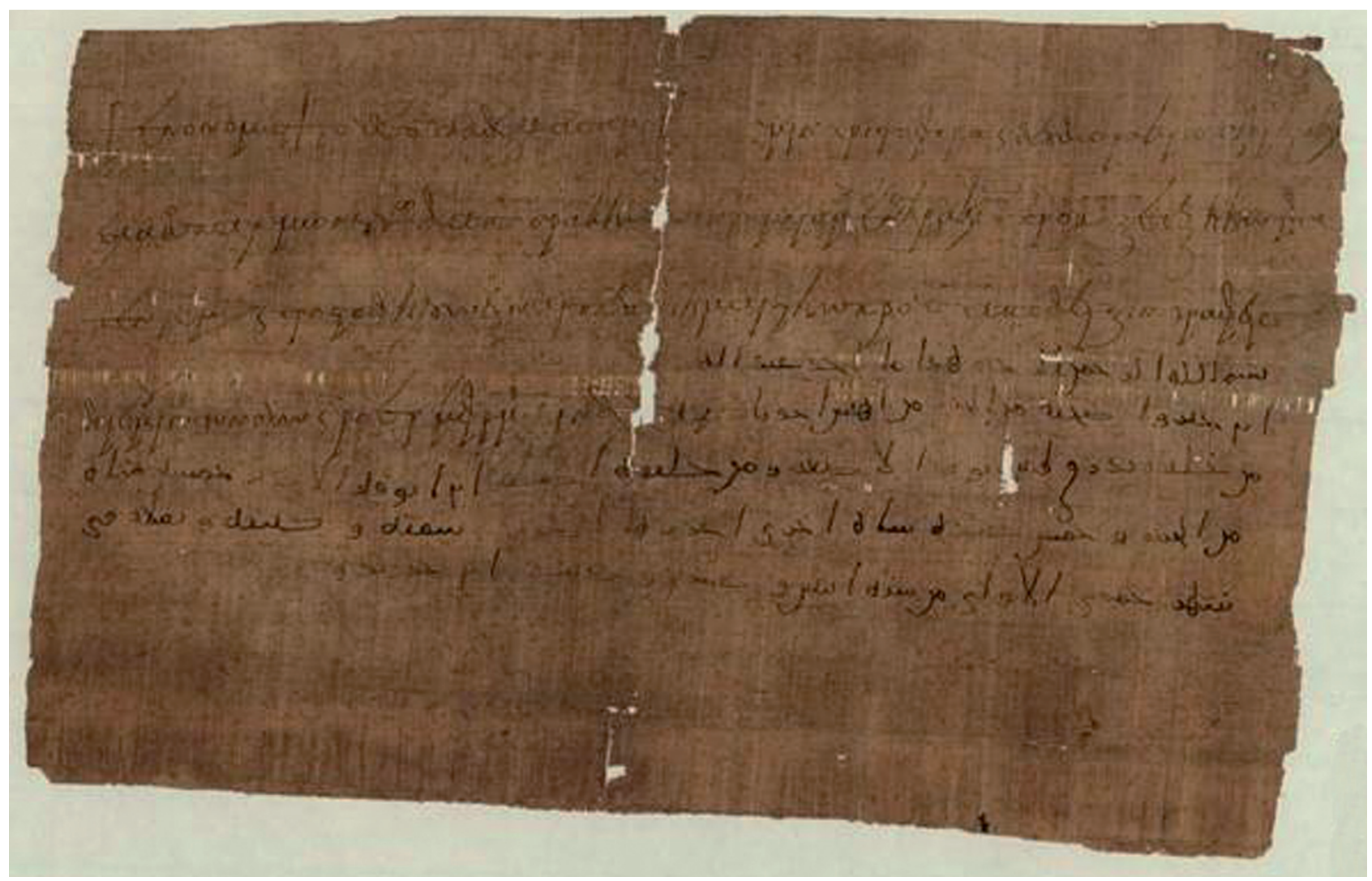

Abb. 1: P. Vindob. G 39726 (griechisch-arabische Empfangsbestätigung des Emirs Abdella über 65 Schafe): Herakleopolites, 643 n. Chr.

arabischen Reiches sind Texte in allen drei Sprachen relevant, doch die Entzifferung und historische Einordnung der oftmals fragmentarischen Schriftstücke ist sehr voraussetzungsreich und braucht viel Zeit. Während zehntausende griechische und koptische Dokumente bereits publiziert vorliegen, ist bislang erst eine kleine Zahl von Papyri in arabischer Sprache ediert worden. Obwohl Sammlungen und Museen in Ägypten, Europa und Nordamerika riesige Bestände an nicht publizierten arabischen Texten auf Papyrus und verwandten Beschreibmaterialien haben, ist die arabische Papyrologie ein nur von einer kleinen Zahl von Spezialisten betriebenes Forschungsfeld.

Die Papyrussammlung der Österreichischen Nationalbibliothek beherbergt mit etwa 180000 Objekten eine der größten Sammlungen antiker Schriftstücke weltweit. Die Objekte kommen - wie die Papyri generell - fast ausschließlich aus Ägypten, wo die klimatischen Bedingungen die Erhaltung der fragilen Objekte ermöglichten. Der Schwerpunkt der Sammlung liegt auf Dokumenten aus der spätantiken und frühmittelalterlichen Epoche (5.-10. Jh. n. Chr.), wobei die umfangreichste Gruppe die Schriftstücke in arabischer Sprache sind: Die ca. 80000 Dokumente sind die größte Sammlung arabischer Papyri und früher Papiere, von denen viele aus den frühen Jahrhunderten (7.-9. Jh. n. Chr.) der Herrschaft der Araber über das Land am Nil stammen. Die Zahl der edierten arabischen Dokumente ist wegen der sowohl paläographischen als auch sprachlichen Schwierigkeiten jedoch sehr niedrig: Nur etwa 900 Dokumente sind bislang publiziert worden, obwohl selbst nach vorsichtigen Schätzungen mindestens 40000 eine Publikation lohnen würden.

Hier setzt das Forschungsprojekt „Papyri of the Early Arab Empire“ an, das seit 2013 von der US-amerikanischen Andrew W. Mellon Foundation gefördert wird: Es hat sich zum Ziel gesetzt, die nicht publizierten arabischen Bestände der Papyrussammlung systematisch zu sichten und 15000 Dokumente auszuwählen, die einerseits von ihrem Inhalt her besonderes Interesse beanspruchen und andererseits nach Erhaltungszustand und Schwierigkeitsgrad für eine baldige Edition geeignet erscheinen. Der Focus soll auf Texten aus den frühen Jahrhunderten der arabischen Herrschaft liegen, um die formative Periode des Reiches unter den Omayyaden und Abbasiden zu studieren. Zugleich werden damit jene historischen Prozesse beleuchtet, welche in langsamer, aber nachhaltiger Veränderung vom christlich-byzantinischen Ägypten schrittweise zum arabisch-islamischen Ägypten hinüberleiteten. Damit wird eine substantielle Zahl von dokumentarischen Quellen, welche als wesentliche Ergänzung oder als Korrektiv $\mathrm{zu}$ den historiographischen Zeugnissen hinzutreten, für 
weiterführende Forschungen zur Verfügung gestellt. Da nahezu alle auf Papyrus und Pergament bzw. (ab dem 9. Jh.) auf frühem Papier geschriebenen Texte aus dem Alltagsleben und der Verwaltung des Landes stammen, beleuchten sie verschiedene Bereiche des privaten und öffentlichen Lebens sowie der staatlichen Herrschaft in Ägypten, das stets ein Kernland des arabischen Reiches gewesen ist. Paradigmatisch wird dabei die Entwicklung eines integrativen Teiles des arabischen Reiches illustriert, wo dank der Papyri sowohl das Wirtschafts- und Rechtsleben als auch die mittleren und unteren Ebenen der Steuerverwaltung und des staatlichen Apparates in einem Umfang dokumentiert sind, der für keinen anderen Teil des früharabischen Reiches vorliegt.

Für die Geschichte der ersten drei Jahrhunderte der arabischen Epoche Ägyptens sind freilich nicht nur die Schriftstücke in arabischer, sondern auch jene in griechischer und koptischer Sprache relevant. Seit der Eingliederung Ägyptens in das Reich Alexanders des Großen (um 331 v. Chr.) war Griechisch als Verwaltungs- und Verkehrssprache der sozialen und administrativen Eliten des Landes etabliert, während die einfache Bevölkerung in den agrarisch geprägten Landstrichen weiterhin Ägyptisch (das man in seiner spätantiken Ausprägung als Koptisch bezeichnet) gesprochen hat. Diese multilinguale Kultur Ägyptens ist eine kulturhistorisch bemerkenswerte Erscheinung, die nach der arabischen Eroberung noch um eine Facette erweitert wurde. Auch nach der Installierung der arabischen Herrschaft blieb Griechisch für mindestens vier Generationen die Sprache der Verwaltung, während Koptisch weiterhin das beherrschende Idiom der einfachen Bauern und Handwerker sowie des christlichen Klerus blieb. Da die Einwanderung arabisch-stämmiger Bevölkerung zunächst nur in sehr beschränktem Umfang stattfand, gewann auch die arabische Sprache erst im Verlauf des 8. und 9. Jh. allmählich an Boden. Die Verlagerung des sprachlichen Schwerpunktes zunächst von Griechisch auf Arabisch, dann von Koptisch auf Arabisch ist in sich ein höchst komplexer und wichtiger Teil der politischen, kulturellen und religiösen Veränderungen. Um diesen Transformationsprozess adäquat erfassen zu können, berücksichtigt das Projekt auch Texte in griechischer und koptischer Sprache. Da die Papyrussammlung auch in diesem Bereich über sehr umfangreiche Bestände verfügt (ca. 60000 griechische Papyri und ca. 35000 koptische Texte) werden diese Gruppen gleichfalls systematisch auf Dokumente aus dem relevanten Zeitabschnitt hin durchgesehen und für eine weitere wissenschaftliche Bearbeitung aufbereitet.

Das Forschungsprojekt „Papyri of the Early Arab Empire“ hat sich zum Ziel gesetzt, aus den nicht publizierten
Beständen der Papyrussammlung eine gezielte Auswahl von Schriftstücken in (vornehmlich) arabischer, griechischer und koptischer Sprache zu treffen und diese über die Webseite der Österreichischen Nationalbibliothek der Öffentlichkeit frei zugänglich zu machen (http:// www.onb.ac.at/sammlungen/papyrus/papyrus_bestands recherche.htm). Von jedem Objekt sind hochaufgelöste digitale Abbildungen zur Ansicht frei gegeben; zudem bietet ein Katalog von Metadaten die wichtigsten Informationen über Herkunft, Inhalt, Datierung und allfällige Besonderheiten oder erwähnenswerte Details jedes Dokuments (etwa die Zugehörigkeit zu einem Textkonvolut, prosopographische oder topographische Daten, paläographische Auffälligkeiten etc.). Da die antiken Schriftstücke nicht einfach $\mathrm{zu}$ lesen und $\mathrm{zu}$ verstehen sind, geben solche Informationen entscheidende Hinweise und Hilfestellungen für eine weitere Erforschung und Rezeption der Texte.

Die wissenschaftlichen Herausforderungen beim Arbeiten an dem Projekt liegen vor allem darin, aus der Fülle von Schriftstücken diejenigen auszuwählen, die einerseits für die Fragestellung und den Zeitabschnitt aussagekräftig, andererseits auch geeignet für eine zügige Bearbeitung sind. Hierfür müssen Tausende Dokumente zumindest partiell entziffert, nach ihrem Inhalt bestimmt und in ihrem Kontext eingeordnet werden. Falls die Dokumente kein Datum tragen, müssen sie aufgrund paläographischer Beobachtungen auch zeitlich eingeordnet werden. Zudem gilt es, Besonderheiten im Urkundentypus, im Formular oder im Inhalt zu erkennen. Dies alles stellt höchste Anforderungen an die Expertise der Mitarbeiter, die durchweg auf eine jahrelange Erfahrung in der Erforschung solcher Schriftstücke zurückblicken können. Falls nötig, müssen die ausgewählten Dokumente auch einer konservatorischen Behandlung unterzogen werden. Vielfach sind die aus dem Sande geborgenen Schriftstücke noch in dem Zustand, in dem sie vor ca. 140 Jahren nach Wien in die Papyrussammlung gelangt sind. Sie müssen von Verschmutzungen gereinigt werden, oftmals sind lose Fasern zu stabilisieren, Fragmente zu sichern und Faltungen zu glätten. Erst nach dieser konservatorischen Aufbereitung können die Objekte zur Digitalisierung gehen. Schließlich werden die digitalen Abbildungen und die Metadaten zusammengeführt und in Form einer OnlineDatenbank, die nach Stichworten abfragbar ist, auf der Webseite frei zugänglich gemacht (open access). Entsprechend der Ausrichtung der Sammlungsbestände gehören die allermeisten Papyri und Papiere den dokumentarischen Schriftstücken des Alltags an; gelegentlich entdeckte Texte literarischen Inhalts (etwa Fragmente früher Koranhandschriften) werden bevorzugt aufgenommen. 


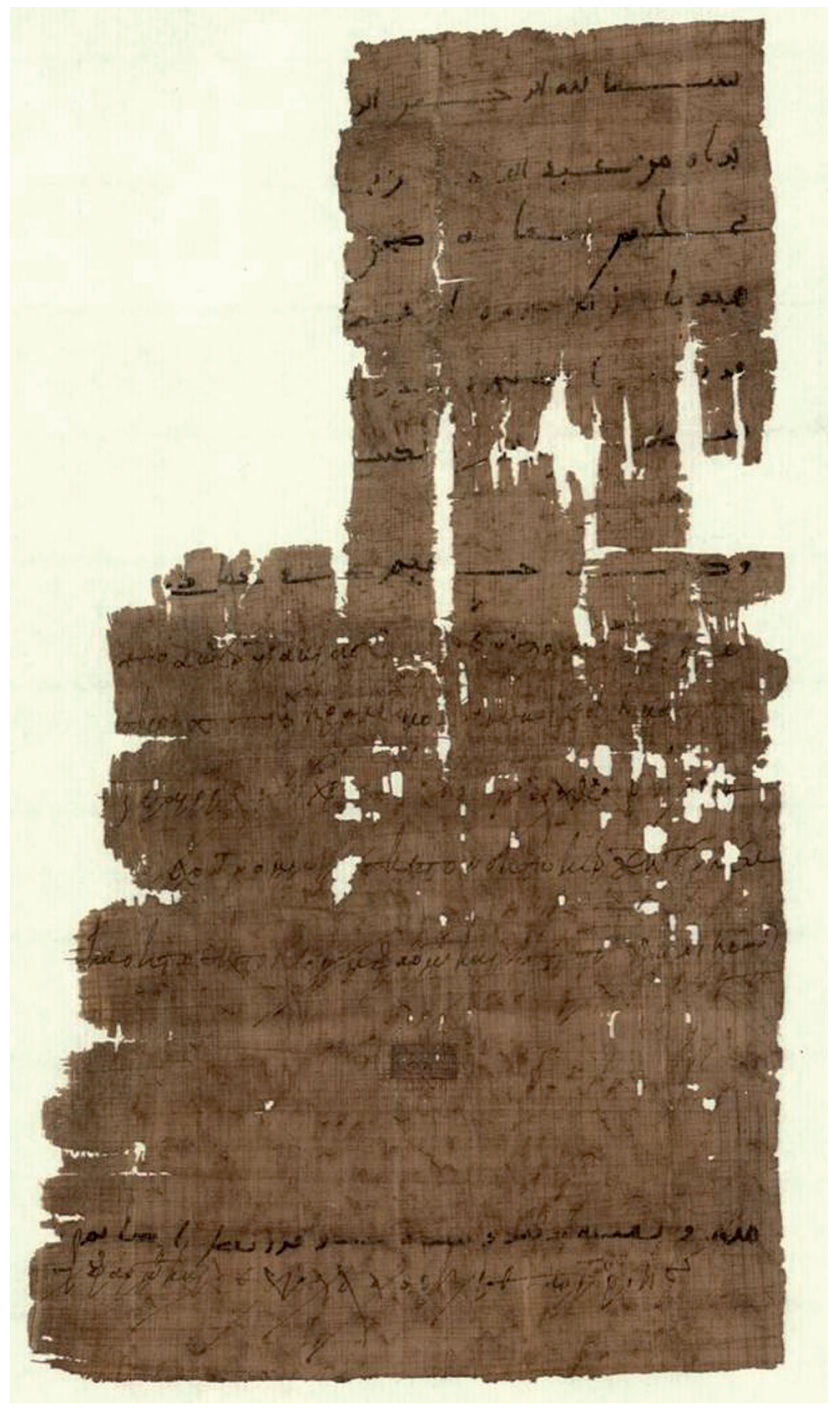

Abb. 2: P. Vindob. G 39738 (griechisch-arabische Abgabenquittung): Herakleopolies, 677 oder 707 n. Chr.

So entsteht im Verlaufe des Forschungsprojektes eine gezielt zusammengestellte, umfangreiche Sammlung digitaler Abbildungen samt erklärender Metadaten zu den antiken Schriftstücken, die als neue Quellen den gelehrten Diskurs über das Früharabische Reich bereichern und nachhaltig beeinflussen werden. Zudem ist dadurch die Voraussetzung geschaffen, dass ein in Anzahl und Inhalt gleichermaßen bedeutender Bestand der Papyrussammlung der Österreichischen Nationalbibliothek für eine voll- ständige, kommentierte Edition und detaillierte historische Auswertung aufbereitet ist. Damit schafft das Projekt eine wesentlich erweiterte Grundlage für laufende und zukünftige Editionsvorhaben und historisch orientierte Forschungen, die an der Papyrussammlung selbst angesiedelt sind oder von internationalen Gelehrten in Kooperation mit der Nationalbibliothek durchgeführt werden. Drehscheibe dieser Forschungen ist die international vernetzte Papyrussammlung, welche nicht nur die antiken Schriftstücke beherbergt und pflegt, sondern auch über eine hervorragende Fachbibliothek verfügt.

Die Intention des Projektes ist es, einerseits das Interesse an den arabischen Dokumenten zu wecken und der Gelehrtenwelt das Potential der riesigen, noch nicht publizierten Bestände vor Augen zu führen, um eine intensivierte Bearbeitung anzuregen. Insbesondere der arabischen Papyrologie sollen durch neues Material entscheidende Impulse gegeben werden. Andererseits verfolgt das Projekt die Absicht, der Forschung eine substantielle Zahl von dokumentarischen Texten bekannt zu machen, die für die kontrovers debattierten Fragen zur Geschichte des Früharabischen Reiches, der Ausbreitung des Islams und des Korans, den Anfang der arabischen Schriftlichkeit, der früharabischen Paläographie, der Organisation arabischer Herrschaft und Staatlichkeit sowie vielfältigen Aspekten der Alltagskultur aussagekräftig sind. Die Digitalisierung von arabischen, griechischen und koptischen Texten aus den ersten Jahrhunderten der arabischen Herrschaft soll Material für künftige Editionen zur Verfügung stellen und damit in weiterer Folge relevante Quelle für weitergreifende historische Fragestellungen zur frühen arabischen Geschichte aufbereiten.

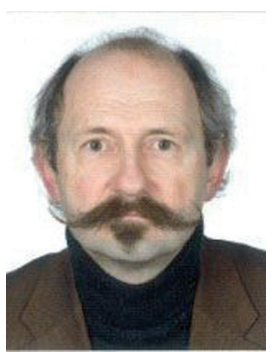

Univ.-Prof. Dr. Bernhard Palme Österreichische Nationalbibliothek Papyrussammlung und Papyrusmuseum Josefsplatz 1 A-1015 Wien Österreich bernhard.palme@onb.ac.at 